\title{
Formulation and Evaluation of Cat Fish Slim Mucin Ointment for Wound Healing
}

\author{
MA Momoh ${ }^{1^{*}}$, SA Brown, ${ }^{2}$ and CC Muogbo ${ }^{1}$ \\ ${ }^{1}$ Drug Delivery Unit, Department of Pharmaceutics, Faculty of Pharmaceutical Sciences, University of Nigeria, Nsukka, ${ }^{2}$ Faculty \\ of Pharmaceutical Sciences, University of Port Harcourt, Nigeria \\ *For correspondence: Email: jointmomoh@yahoo.com; Tel: +234-8037784357
}

Received: 3 August 2012

Revised accepted: 3 October 2013

\begin{abstract}
Purpose: To evaluate the effect of fish mucin ointment on wound healing in a rat model.

Methods: Fish mucin was formulated into an ointment using soft paraffin ointment base. Its woundhealing activity and toxicity were evaluated using an incision and excision wound model in rats. A range of concentrations $(2.5-10 \% \mathrm{w} / \mathrm{w})$ of mucin in the ointment was tested to determine the concentration that will give optimum wound healing activity The ointment base (i.e., without mucin) was used as control while $2 \%$ nitrofurazone served as positive control.

Results: Wound healing activity increased with increase in mucin concentration up to a maximum of 5 $\%$. Negative control produced wound healing of $41.2 \pm 0.0 \%$ on the $19^{\text {th }}$ day with the mean hydroxyproline content and tensile strength being $682.07 \pm 0.11 \mu \mathrm{g} / \mathrm{g}$ and $874.11 \pm 0.39 \mathrm{~g} / \mathrm{cm}^{2}$, respectively. Mucin ointment (5\%) exhibited $100 \%$ wound healing properties on day 12 . Significant increase $(p>0.05)$ in skin tensile strength $\left(1311.02 \pm 0.16 \mathrm{~g} / \mathrm{cm}^{2}\right)$ and hydroxyproline $(1163.11 \pm 0.16$ $\mu \mathrm{g} / \mathrm{g}$ ) was found for mucin ointment as against $1151.21 \pm 0.41 \mathrm{~g} / \mathrm{cm}^{2}$ and $875.12 \pm 0.84 \mu \mathrm{g} / \mathrm{g}$, respectively, for positive control. At the concentrations tested, no toxicity was recorded for mucin ointment.

Conclusion: This study demonstrates that the fish mucin ointment promotes wound-healing.
\end{abstract}

IKeywords: Fish mucin, Ointment, Wound, biomaterial.

Tropical Journal of Pharmaceutical Research is indexed by Science Citation Index (SciSearch), Scopus, International Pharmaceutical Abstract, Chemical Abstracts, Embase, Index Copernicus, EBSCO, African Index Medicus, JournalSeek, Journal Citation Reports/Science Edition, Directory of Open Access Journals (DOAJ), African Journal Online, Bioline International, Open-J-Gate and Pharmacy Abstracts

\section{INTRODUCTION}

Wound healing is an intricate process in which the skin (or another organ-tissue) repairs itself after injury. Without the ability to heal wounds, the body will succumb to hemorrhage or infection. In the last two to three decades, there has been a revolution in wound management. Since the discovery of the first growth factor in 1962 - epidermal growth factor - the science of wound repair and regeneration has advanced enormously [1]. Other research has prompted the change from the outdated 'dry dressing technique' to the moist wound concept of healing
[1]. These two major advances have led to a rethinking of the management of wound repair and to an exponential growth in the development of new wound dressings. Unfortunately, the management of both acute and chronic wounds are inconsistent and thus wounds consume an inordinate amount of patient money in the poor resources country like Nigeria [2]. Wound care practices can and must be optimised. However, progressive wound management is not inextricably determined by the use of modern wound dressings. 
Mucins are a family of large glycosylated proteins (50\% w/w carbohydrate). Mucins are group of nitrogenous substances secreted by a mucous gland. Snail mucin has been evaluated for wound healing, gastroprotective $[3,4]$ and a carrier for peptide drug [5]. It is of scientific and pharmaceutical interest to also evaluate mucin from aquatic animals such as fish. The mucus of some cat fishes have shown pronounced activities in biomedicine [6]

In order to facilitate cost reduction and also to improve the wound healing process, alternative source of wound healing need to be explored. The aim of this study is to evaluate the wound healing activity of cat fish slim mucin formulated into an ointment for topical application.

\section{EXPERIMENTAL}

\section{Materials}

Acetone (BDH Chemicals); Diazepam injection (GlaxoWellcome), methylated spirit (Hardis and Dromedras) and distilled water were from an all glass still. The fish mucin was obtained from a batch prepared in our laboratory following earlier established procedures $[7,8]$.

\section{Collection of fish mucin}

The animal experimental protocols were approved by our institution's Animal Ethics Committee and were in compliance with the Federation of European Laboratory Animal Science Association and the European Community Council Directive of November 24, 1986 (86/609/EEC) [10].

Mucus was collected as described by Momoh et al [8] with slight modifications. Mucus was obtained from 20 catfish from a fish dealer in a local market. Before collecting epidermal mucus, the fish were kept in a make shift running water and maintained on fish diet for five days and then electrocuted by placing them in a small quantity of water in a bowl and passing current to the water directly. Mucus was carefully scraped from the dorsal body surface using a plastic spatula. The aqueous collections were pooled together and subjected to further purification by precipitating with chilled acetone and then dried by lyophilization (Labconco Freezone apparatus, USA). Briefly, the catfish mucin mucilage was rapidly frozen in a $95 \%$ ethanol bath at $-25{ }^{\circ} \mathrm{C}$ for $25 \mathrm{~min}$ and then placed in a vacuum chamber of the lyophilizer (Labconco Freezone apparatus, USA). Lyophilization was carried out at $-25{ }^{\circ} \mathrm{C}$ under vacuum (1.2 - 3.0 mbar) for $24 \mathrm{~h}$. The greyishbrown lyophilized flakes of the fish mucin were collected and pulverized into fine powder using a mortar and pestle, was and then stored at $-25{ }^{\circ} \mathrm{C}$ until used in this study.

\section{Ointment preparation}

Simple ointment containing the mucin extracted from cat fish was prepared by trituration method in a ceramic mortar and pestle using soft paraffin ointment base. Four different concentration of the ointment $(2.5,5.0,7.5$ and $10.0 \% \mathrm{w} / \mathrm{w})$ of the fish mucin were prepared for this study. Nitrofurazone ointment $(0.2 \% \mathrm{w} / \mathrm{w})$ was used as standard for comparing wound healing potential of mucin in different animal model. The ointments were packed in a plastic container and store in the refrigerator until they were used.

\section{Experimental rats}

Fourty two albino rats of $120.00 \mathrm{~g} \pm 0.21$ mean body weight were used in this study. The animals were procured from the animal house of Department of Pharmacology and Toxicology, University of Nigeria, Nsukka, Nigeria, and were divided into two groups (1 and 2); each group was further sub-divided into four groups $(A-F)$ of three rats each and kept in separate metallic cages and maintained under standard housing conditions (room temperature $24-27^{\circ} \mathrm{C}$ and humidity 60 - $65 \%$ with 12 h light/dark cycle. Food was provided in the form of dry pellets and water ad libitum. Animals in group 1 were used for the excision model, while those in group 2 were used for the incision model study.

\section{Preparation of wound site in experimental animals}

Wound healing studies were based on two models - excision and incision.

\section{Excision wound model}

The wound site was prepared following the incision wound model [9]. The albino rats were anaesthetized with diazepam $(0.2 \mathrm{mg} / \mathrm{kg}$ body weight) and the hairs on the skin of the animals' back were shaved with a sterilized razor blade. A circle of diameter $20 \mathrm{~mm}$ was marked on each right side of the thigh of the animal's skin surface, and the skin dissected out. The area was measured immediately by tracing out the wound area using a transparent tracing paper and the squares counted and the area recorded. 


\section{Determination of the rate of wound healing}

Treatment was initiated immediately after the incision was made by applying ointments topically on the wound and then once in every two days. All the ointments were applied topically using sterile cotton wool. The wound area of each animal was measured using a millimetrescale graph paper while the animals were under mild anesthesia on the days following post surgery. Each application was evaluated in five rats per group and the results shown as a mean of five determinations.

\section{Incision wound model}

Animals in group 2 (A-F) were used for the study. All the animals were anesthetized with diethyl ether. Paravertebral incision of $6 \mathrm{~cm}$ long was made on either side of vertebral column of rat. Care was taken to make the incision at least $1 \mathrm{~cm}$ lateral to vertebral column. The wounds were covered with interrupted suture of $1 \mathrm{~cm}$ apart and treated as follows: group A served as negative control and was treated with ointment base (without drug), group B was reference standard (positive control) and treated with 0.2 $\% w / w$ nitrofurazone ointment. Subgroups $\mathrm{C}-\mathrm{F}$ were treated with graded concentrations of the ointments (2.5, 5.0, 7.5 and $10.0 \%)$, respectively. The animals were caged separately according to thier groups. The sutures were removed after the $8^{\text {th }}$ day of surgery.

\section{Tensile strength measurement}

The tensile strength of wound was measured after the 10th day of surgery using an established method (11). Briefly, the alternate sutures were removed on $8^{\text {th }}$ day and on the ninth day the remaining suture was removed and tensile strength measured on the 10th day. Rats were anesthetized as described earlier and strips of healing tissue along with normal skin at the two ends were excised. Tensile strength was measured by loading a strip between the upper and lower holders of the tensiometer (NK-T 234, UK). The amount of load that was able to break the healed wound was recorded and converted to tensile strength $(T)$ using Eq 1.

$T=L / A$

where $L$ is the total beaking load and $A$ is the cross-sectional area of the healed wound

\section{Determination of hydroxyproline content}

Hydroxyproline content was analyzed using acid hydrolysis method [11]. Granulation tissues were extracted in $5 \%$ trichloroacetic acid (TCA) and incubated overnight with $10 \%$ perchloric acid. It was centrifuged after incubation and washed with $5 \%$ perchloric acid. The precipitate was dissolved in warm $10 \%$ perchloric acid and the hydroxyproline content was estimated. Briefly, healed tissues of the wound area were cut and dried in a hot air oven at $70-75{ }^{\circ} \mathrm{C}$ until a constant weight was attained; the dried material was and hydrolyzed in $6 \mathrm{M} \mathrm{HCl}$ at $130{ }^{\circ} \mathrm{C}$ for $3 \mathrm{~h}$ in sealed tubes. The resultant hydrolyzate was neutralized to $\mathrm{pH} \quad 7.0$, and chloramine-T oxidation test was carried out on the hydrolyzate within 20 min. Perchloric acid (0.4M) was added to terminate the reaction and color was developed with the help of Ehrlich reagent at 60 ${ }^{\circ} \mathrm{C}$ and measured spectrophotometrically (Jenway, 6405, UK) at $545 \mathrm{~nm}$.

\section{Acute toxicity study}

Swiss albino mice of either sex weighing (20 - 22 g) and 4 weeks old were used for acute oral toxicity study. The study was carried out as per the guidelines set by OECD [12]. The animals were starved overnight and divided into six groups $(n=3)$. They were fed with increasing doses $(100,150$ and $200, \mathrm{mg} / \mathrm{kg}$ ) mucin using intubation tube. The animals were continuously observed for mortality and behavioural responses for $48 \mathrm{~h}$ and thereafter daily for 2 weeks after administration.

\section{Statistical analysis}

The results were expressed as mean of SEM. Statistical analysis of the data was carried out using SPSS statistical package, version 16.0 (SPSS Inc. Chicago, IL, USA) and Student's ttest to determine where there were statistically significant differences $(p \leq 0.05)$.

\section{RESULTS}

The mean closure of wound area (\%) was examined every 3 days up to 18 post-wound days and taken as a measure of wound healing. The results (Table 1 ) for excision wound model showed significant improvements in all measured parameters. There was a progressive decrease in wound radius of the animals with time. By the 12th day, the wound area of all the animals treated with mucin ointment showed more than $80 \%$ healed with the exception of $5 \%$ formulation which showed $100 \%$ healed as compared with the control groups. The ointment $(5.0 \%)$ treated (group D) showed faster epithelialisation $(12.1 \pm 0.1)$ than the animals treated with $2.5,7.5$ and $10.0 \%$ ointment, respectively. The period of epithelialisation was $19.12 \pm 0.32$ in the case of positive control ( $2 \%$ 
Table 1: Wound healing activity of the formulations in experimental rats

\begin{tabular}{lccccccc}
\hline Group & \multicolumn{2}{l}{ Wound healing (\%) } & \multicolumn{7}{l}{} \\
\cline { 2 - 6 } & Day 1 & Day 3 & Day 6 & Day 9 & Day 12 & Day 15 & Day 19 \\
\cline { 2 - 6 } A (NC) & 0.0 & $4.9 \pm 3.1$ & $12.3 \pm 2.0$ & $30.1 \pm 0.1$ & $31.6 \pm 3$ & $34.4 \pm 1.2$ & $41.2 \pm 0.0$ \\
B (PC) & 0.0 & $15.0 \pm 2.0$ & $28.3 \pm 2.1$ & $47.1 \pm 0.2$ & $53.1 \pm 0$ & $76.0 \pm 1.0$ & $96.3 \pm 1.0$ \\
C (2.5\%) & 0.0 & $27.5 \pm 2.1$ & $56.4 \pm 0.0$ & $76.3 \pm 3$ & $88.4 \pm 4$ & $97.2 \pm 2.2$ & - \\
D (5.0\%) & 0.0 & $33.3 \pm 0.0$ & $65.3 \pm 4.0$ & $88.8 \pm 2.3$ & $100 \pm 0.0$ & - & - \\
E (7.5\%) & 0.0 & $26.2 \pm 4.0$ & $44.2 \pm 2.1$ & $75.3 \pm 5.0$ & $86.7 \pm 1.0$ & $100.0 \pm 0$ & - \\
F (10\%) & 0.0 & $29.3 \pm 0.0$ & $57.3 \pm 0.0$ & $72.8 \pm 2.3$ & $87.5 \pm 2.0$ & $96.1 \pm 1.0$ & - \\
\hline
\end{tabular}

Values are given as mean \pm S.D; $N C=$ negative control; $P C=$ positive control

w/w nitrofurazone ointment). Physical examination showed a smooth surface area of the healed site in all the formulation. In incision wound model, the rats treated with mucin formulations $(2.5,5.0,7.5$ and $10.0 \%)$, showed increased $(p<0.005)$ breaking strength of $1062.11 \pm 0.03,1311.02 \pm 0.16,1128.08 \pm 0.15$ and $1104.01 \pm 0.13 \mathrm{~g} / \mathrm{cm}^{2}$, respectively, compared with negative control (874.11 \pm 0.39 $\mathrm{g} / \mathrm{cm}^{2}$ ). The mean breaking strength was also significant in animals treated with standard drug nitrofurazone ointment $\left(1151.21 \pm 0.43 \mathrm{~g} / \mathrm{cm}^{2}\right)$. The highest breaking strength was obtained with $5.0 \%$ mucin formulation (Table 2 ).

Hydroxyproline content data (Table 2) revealed that the animals (subgroups C, D, E and F) treated with increasing concentration of mucin ointment showed higher hydroxyproline content than positive control (2\% nitrofurazone) with subgroup $D$ showing the highest content $(1163.11 \pm 0.16 \mu \mathrm{g} / \mathrm{g})$. Negative control group (ointment base) showed the least hydroxyproline content $(682.07 \pm 0.11 \mu \mathrm{g} / \mathrm{g})$.

Toxicological evaluation revealed no sign of death or any abnormality in the behaviour of the rats in all the groups at all doses tested. Normal feeding and water intake by the rats was restored 3 days after the initial trauma as a result of the inflicted wound. Animals in NC group showed poorer recovery rate, and this may be attributed to the non-application of medicament to the wound area.

\section{DISCUSSION}

The term, 'mucus', applies to substances in which carbohydrates constitute a predominant proportion (mostly $60 \%$ or more). The epidermal gel secretion of cat fish is unlike what can be generally termed mucus. Over $85 \%$ of the dry weight of the gel secretion is protein, with lipids (13.4 \% of the dry weight) and only small amounts of carbohydrates and nucleic acids [6].The lipid fraction contains a relatively high level of arachidonic acid which is a twentycarbon atom and has been implicated in maintaining the integrity of gastrointestinal tract a mixture of eicosanoids, cholesterol and cholesteryl esters, as well as polar lipids [13]. The proteins are composed of two major fractions, the water-insoluble a-helical structures and the phosphate buffer-soluble fraction [13].

Healing of wound is a complex phenomenon involving various phases, coagulation, inflamemation, collagenation, wound contraction, epithalialization and remodeling. The phases between coagulation and collagenation are intimately related while those of wound contraction and epitheliazation are independent of each other but run concurrently [14]. Drugs, which influence one phase, may not necessarily influence another. Hence, different models have been used in our study to assess the effect of various phases, which run concurrently, but independent of each other. The results of present study showed that the ointment formulated with mucin extracted from fish possesses definite healing activity. This is demonstrated by a significant increase in the rate of wound contraction and by enhanced epithelization. Significant increase was also observed in skin breaking strength and hydroxyproline content which is a reflection of increased collagen levels due increased crosslinking of collagen fibres.

Table 2: Hydroxyproline content and tensile strength of healed wound tissue (mean \pm SD)

\begin{tabular}{lcc}
\hline Group & Hydroxyproline $(\boldsymbol{\mu g} / \mathbf{g})$ & Tensile strength $\left(\mathbf{g} / \mathbf{c m}^{2}\right)$ \\
\hline (A) Ointment without mucin & $682.07 \pm 0.11$ & $874.11 \pm 0.39$ \\
(B) $2 \%$ nitrofurazone & $875.12 \pm 0.84$ & $1151.21 \pm 0.41$ \\
(C) $2.5 \%$ ointment & $1002.03 \pm 0.22$ & $1062.11 \pm 0.03$ \\
(D) $5.0 \%$ ointment & $1163.11 \pm 0.16$ & $1311.02 \pm 0.16$ \\
(E) $7.5 \%$ ointment & $968.04 \pm 0.24$ & $1128.08 \pm 0.15$ \\
(F) $10.0 \%$ ointment & $910.12 \pm 1.19$ & $1104.01 \pm 0.13$ \\
\hline
\end{tabular}


The process of wound healing moght have been aided by the adhesive nature of mucin, and being a biological substance, it facilitates intimate contact of the healing material with the wound surface. Moreover, it has been observed that mucin generally has adequate wound healing properties [15]. Snail mucin has also been reported to heal wounds $[7,15]$.

It was observed in this study that fish mucin promotes wound healing and regeneration of hairs. Fish mucin played an important role in wound healing as it enhances skin natural regenerative response, probably through immune response. The smoothness observed in animals treated with the mucin formulations indicates that the preparation may prevent hypertrophic scar often called kelloid [7,15]. Several studies have convincingly established that repeated application of preparations involving catfish epidermal secretions to wounds and persistent diabetic foot ulcers in human subjects had a positive effect on wound healing [16]. The complex mixture of components in the epidermal secretion apparently induces a balanced stimulation of the early stages of wound healing. Despite intensive research, the exact nature and role of the wound healing components secreted by club cells are not known, although synergistic effects by the different biologically active components are expected. Our observations during treatment of wound models using preparations (mucins) from the skin of the catfish showed that healing progressed smoothly more than the antibiotictreated group (positive control).

In our earlier work, we reported the bioadhesive properties of mucin [17]. The present study confirms its bioadhesive properties. It also shows that it has better wound healing activity than snail mucin [17]. This may be due to the action of unique components present in fish. Fish mucin has been shown to have antimicrobial properties [18], and this is probably a contributory factor to its wound healing activity observed in this study. Biologically, the interface between fish and its aqueous environment consists of a mucus layer composed of diverse biochemical secretions from epidermal and epithelial cells which may serve as a protective barrier against potential pathogens of their environment [18]. Previous reports of the protective role of mucus and its components in various fish species suggest that the epidermal mucus acts as a first line of defense against pathogens and therefore may offer a potential source of novel antimicrobial components [19] Conversely, the negative effects of bacterial in wound healing cannot be ignored; hence the antibacterial activity of the fish mucin may be a boost to its wound healing potential.

The likely mechanism of fish mucin wound healing action, apart from its antibacterial effect, might also have resulted from the oxidative properties of fish mucin as reported by an earlier researcher who indicated that oxidative stress and free radicals are implicated in impaired wound healing [19]. Molecular oxygen plays a central role in the pathogenesis and therapy of chronic wounds. Overproduction of reactive oxygen species (ROS) results in oxidative stress thereby causing cytotoxicity and delayed wound healing. Therefore, elimination of ROS could be an important strategy in wound healing. All this process will lead to an increase in the intracellular communication in cultured fibroblasts, hence induces a more rapid maturation of granulation tissue. There was no sign of adverse or side effects recorded. This observation was in agreement with our earlier study $[12,16]$. We therefore conclude that at the dose tested the mucin was highly tolerated by the animal.

\section{CONCLUSION}

Cat fish mucin ointment has remarkable wound healing potentials with formulation containing 5.0 $\%$ mucin exhibiting optimum wound healing activity. The mucin was not toxic to the animals at the concentrations tested. Thus, our findings have opened a new window for the development of alternative wound healing products.

\section{REFERENCES}

1. Winter GD. Formation of the scab and the rate of epithelization of superficial wounds in the skin of the young domestic pig. Nature 1962; 193: 293-294.

2. Gruen RL, Chang $S$, MacLellan DG. Optimizing the hospital management of leg ulcers. Aust NZJ Surg 1996; 66: 171-174.

3. Cortfield A, Longman R, Sylvester P, Arul S, Myerscough $N$, Pigatelli M. Mucins and mucosal protection in the gastrointestinal tract: new prospects for mucins in the

pathology of gastrointestinal diseases Gut 2002; 47: 594-598.

4. Mortazavi SA, Carpenter BG, Smart JD. Comparative study on the role played by mucus glycoprotein in the rheological behaviors of the mucoadhesive/mucosal interaction. Int. J Pharm 1992; 94: 195-201.

5. Momoh MA, Adikwu MU, Ugwu AA. Insulin-Loaded MucinEudragit Complexation: A Rectal Delivery Approach. J Pharm Res 2012; 5(4): 2052-2056.

6. Pinky SS, Mittal AK. Glycoproteins in the epithelium of lips and associated structures of a hill stream fish Garra lamta (Cyprinidae, Cypriniformes): a histochemical investigation. Anat. Histol. Embryol. 1988; 37: 101-113.

7. Adikwu MU, Mucins and their potentials (editorial). Trop. J. Pharm. Res. 2006; 5: 581-582. 
8. Momoh MA, Adikwu MU. Evaluation of oral insulin delivery using bovine mucin in non-diabetic rats. Journal of pharmaceutical research. 2008; 7: 5-9

9 Momoh MA, Kenechukwu FC, Agboke AA, Adedokun M, Akueyinwa LU. Assessment of wound healing activity of mucinated-Occimum gratissimum powder. J Pharm Res 2012; 5(4), 2061-2064

10. European Community Council Directive on the ethics of experiments involving laboratory animals (86/609/EEC), November 24, 1986.

11. Saha K, Mukherjee PK, Das J, Pal M, Saha BP. Wound healing activity of Leucas lavandulaefolia Res. J Ethnopharmacol 1997; 56: 139-144.

12. Nwokoro EA, Nwanjo HU. Antidiabetic and biochemical effects of extract of Vernonia amygdalina in diabetic rats. J Innov Life Sci 2004; 7: 6-10.

13. Al-Bow HA, Al-Hassan JM, Thomson M, Thulesius $O$, Elkhawad $A$. Multiple vasoactive factors in epidermal secretions of the Arabian Gulf Catfish Arius bilineatus (Valenciennes). Gen Pharmacol 1997; 28: 737-744.
14. Prudden JF, Allen J. The clinical acceleration of healing with a cartilage preparation; a controlled study. JAMA 1965; 192: 352-356.

15. Adikwu MU, Alozie BU. Application of snail mucin dispersed in detarium gum gel in wound healing. Sci Res Ess 2007; 2(6): 195-198.

16. Al-Hassan JM. Diabetic ulcer healing preparations from the skin of the Arabian Gulf catfish (Arius bilineatus val.): a novel and effective treatment. Int $J$ Tiss React 1990; 12: 121-135.

17. Momoh MA, Adikwu MU, Eraga SO. The Effect of Cast Films of Bovine Mucin and Prosopis Gum Containing a Cicatrin® Powder on Wound Healing in rats. Dhaka University J Pharm Sci 2008; 7: 710.

18. Bradshow CM, Richard AS, Sigel MM. IgM antibodies in fish mucus. Soc. exptl. Biol. Med. Proc . 1971; 136: 1122-1124.

19. Huang $X$, Li $Q$, Li $H$, Guo L. Neuroprotective and antioxidative effect of cactus polysaccharides in vivo and in vitro. Cell Mol Neurobiol 2009; 29(8): 1211-1221. 